\title{
A Simple Strategy for Sensorless Speed Control for an IPMSM During Startup and Over Wide Speed Range
}

\author{
Hyun-Woo Sim*, June-Seok Lee* and Kyo-Beum Lee ${ }^{\dagger}$
}

\begin{abstract}
This paper presents a hybrid sensorless control for an interior permanent magnet synchronous motor (IPMSM) for zero-, low-, and high-speed regions. Many sensorless control methods such as an observer-based estimator have been introduced. However, most of the observerbased estimators have a disadvantage at start-up and in the low-speed region. To solve this problem, a simple strategy of using a hybrid system is proposed by integrating a high-frequency (HF) signal injection method and a full-order flux observer. In addition, an HF signal injection method with only a low pass filter (LPF) is proposed to simplify the hybrid system. The hybrid system achieves highperformance drive throughout the entire speed range. The effectiveness of the proposed hybrid technique is verified by experiments using an 11-kW IPMSM drive system.
\end{abstract}

Keywords: Permanent magnet synchronous motor, Sensorless control, Signal injection, Full-order observer

\section{Introduction}

Permanent magnet synchronous motors (PMSMs) have high efficiency because there is no field winding in the rotor for the generation of the magnetic flux. Additionally, PMSMs have better high-power density and a faster transient response than a de motor or an induction motor. Because of these characteristics, PMSMs are widely used in general industrial fields, especially in high-performance motor control applications. Therefore, more sophisticated control is required. For effective torque control and speed control, information on the rotor position and speed is necessary. This information is obtained by sensors such as an encoder and a resolver. In the industrial field, however, eliminating sensors is desirable because of the costs associated with sensor faults $[1,2]$. Hence, many sensorless vector control methods have been investigated, such as model-based methods and adaptive flux observers [3-11].

In general, a model-based estimator has been used to estimate the stator flux. This method exhibits high performance for sensorless control. The observer-based estimator has several advantages; namely, it does not assume steadystate conditions, and the stability of the estimate can be analyzed. In particular, a full-order observer is not sensitive to signal distortion and measured noise caused by sensors. Additionally, this estimator is well known to have high performance [5-11]; however, it is not suitable for zeroand low-speed operation because the very small current and back-EMF are generated. Therefore, the high-frequency

\footnotetext{
$\dagger$ Corresponding Author: Dept. of Electrical and Computer Engineering, Ajou University, Suwon, Korea. (kyl@ajou.ac.kr)

* Dept. of Electrical and Computer Engineering, Ajou University, Suwon, Korea. (bigle@ajou.ac.kr, junpb@ajou.ac.kr)

Received: December 11, 2013; Accepted: April 13, 2014
}

(HF) signal injection method is introduced. Because the HF signal injection method does not estimate the current and back-EMF, it exhibits great performance at startup and in the low-speed region [12-16]. In contrast to the back-EMF and observer-based methods, the performance becomes worse when the motor operates at a high speed. To compensate for these problems, hybrid sensorless control methods have been investigated for a wide speed range, including zero speed. For sensorless control of a PMSM, a hybrid structure combines an HF signal injection method and a flux observer using speed-dependent linear functions to smooth transitions. However, conventional methods should determine the switching point that is variable according to load condition and performance of both estimators [17-21]

This paper proposes a hybrid system for interior PMSM (IPMSM) sensorless control, which combines the HF signal injection and full-order observer methods. The HF signal injection method is used for sensorless control for zeroand low-speed operation, whereas a full-order observer is used in the medium- and high-speed regions. The HF signal generates signal injection losses and acoustical noise [18]. The proposed hybrid method determines the optimal switching point by using the error signal of both estimators. When the error signal of both estimated rotor position is close to zero, transition occurs between them. Therefore, stable and smooth transition is guaranteed and it does not need to determine switching speed according to operation and load conditions. In addition, the simple method of HF signal injection is used for decreasing the usage of the filters to reduce the complexity of the algorithm because conventional HF signal injection methods require many filters to obtain the desired rotor position and speed.

The organization of this paper is as follows. A simple 
method of HF signal injection is introduced in Section II. Section III reviews the full-order flux observer. The proposed hybrid system is presented in Section IV. Finally, experimental results demonstrate the validity of the proposed method in Section V.

\section{Simple Method for High-Frequency Voltage Injection}

The general HF injection methods use many filters such as a band pass filter (BPF) and low pass filter (LPF) to eliminate the fundamental frequency and extract the HF signal and rotor position [4, 13-15]. As the number of filters increases, the time delay also increases, which causes an error in the estimated rotor position. In this section, a simple method of HF signal injection is proposed to reduce the number of filters. This method uses a synchronous reference frame transformation with a different variable, making the BPF unnecessary.

\subsection{IPMSM model for HF voltage injection}

The voltage equation of an IPMSM in the synchronous reference frame is defined as follows:

$$
\begin{gathered}
{\left[\begin{array}{l}
v_{d}^{e} \\
v_{q}^{e}
\end{array}\right]=\left[\begin{array}{cc}
R_{s} & 0 \\
0 & R_{s}
\end{array}\right]\left[\begin{array}{l}
i_{d}^{e} \\
i_{q}^{e}
\end{array}\right]+\left[\begin{array}{cc}
p & -\omega_{r} \\
\omega_{r} & p
\end{array}\right]\left[\begin{array}{l}
\lambda_{d}^{e} \\
\lambda_{q}^{e}
\end{array}\right]} \\
{\left[\begin{array}{l}
\lambda_{d}^{e} \\
\lambda_{q}^{e}
\end{array}\right]=\left[\begin{array}{cc}
L_{d} & 0 \\
0 & L_{q}
\end{array}\right]\left[\begin{array}{l}
i_{d}^{e} \\
i_{q}^{e}
\end{array}\right]+\left[\begin{array}{c}
\varphi_{f} \\
0
\end{array}\right]}
\end{gathered}
$$

Where $v_{d q}^{e}, i_{d q}^{e}$, and $\lambda_{d q}^{e}$ are the $\mathrm{d}-\mathrm{q}$ axis voltage and current and the stator flux in the synchronous reference frame, respectively. $R_{s}$ is the stator resistance, and $L_{d q}$ is the stator d-q axis inductance. $\omega_{r}$ is the speed of the rotor in terms of the electric angle, $\varphi_{f}$ is the permanent magnet flux linkage, and $\mathrm{p}$ is the differential operator.

If the rotor angular speed is significantly smaller than the frequency of the injected signal, it can be ignored because the time derivative terms of the currents are proportional to the frequency of the injected signal. Therefore, the voltage equation for the injected HF signal is expressed as

$$
\begin{gathered}
{\left[\begin{array}{c}
v_{d h f}^{e} \\
v_{q h f}^{e}
\end{array}\right]=\left[\begin{array}{cc}
R_{s} & 0 \\
0 & R_{s}
\end{array}\right]\left[\begin{array}{l}
i_{d h f}^{e} \\
i_{q h f}^{e}
\end{array}\right]+\left[\begin{array}{ll}
p & 0 \\
0 & p
\end{array}\right]\left[\begin{array}{l}
\lambda_{d h f}^{e} \\
\lambda_{q h f}^{e}
\end{array}\right]} \\
{\left[\begin{array}{c}
\lambda_{d h f}^{e} \\
\lambda_{q h f}^{e}
\end{array}\right]=\left[\begin{array}{cc}
L_{d} & 0 \\
0 & L_{q}
\end{array}\right]\left[\begin{array}{l}
i_{d h f}^{e} \\
i_{q h f}^{e}
\end{array}\right]+\left[\begin{array}{c}
\varphi_{f} \\
0
\end{array}\right]}
\end{gathered}
$$

where $v_{d h f}^{e}, v_{q h f}^{e}, i_{d h f}^{e}$, and $i_{q h f}^{e}$ are the d-q axis voltages and currents included $\mathrm{HF}$ signal in the synchronous reference frame.

The voltage and stator flux equations are transformed into the stator reference frame as follows:

$$
\begin{aligned}
{\left[\begin{array}{c}
v_{d h f}^{s} \\
v_{q h f}^{s}
\end{array}\right]=} & {\left[\begin{array}{cc}
R_{s} & 0 \\
0 & R_{s}
\end{array}\right]\left[\begin{array}{l}
i_{d h f}^{s} \\
i_{q h f}^{s}
\end{array}\right]+\left[\begin{array}{cc}
p & 0 \\
0 & p
\end{array}\right]\left[\begin{array}{l}
\lambda_{d h f}^{s} \\
\lambda_{q h f}^{s}
\end{array}\right] } \\
{\left[\begin{array}{c}
\lambda_{d h f}^{s} \\
\lambda_{q h f}^{s}
\end{array}\right]=} & {\left[\begin{array}{cc}
L-\Delta L \cos \left(2 \theta_{r}\right) & -\Delta L \sin \left(2 \theta_{r}\right) \\
-\Delta L \sin \left(2 \theta_{r}\right) & L+\Delta L \cos \left(2 \theta_{r}\right)
\end{array}\right]\left[\begin{array}{l}
i_{d h f}^{s} \\
i_{q h f}^{s f}
\end{array}\right] } \\
& +\varphi_{f}\left[\begin{array}{c}
\cos \theta_{r} \\
\sin \theta_{r}
\end{array}\right]
\end{aligned}
$$

Where $L=\frac{L_{q}+L_{d}}{2}, \Delta L=\frac{L_{d}-L_{q}}{2}$, and $\theta_{r}$ is the rotor position.

The HF voltage injection in the stator reference frame is given by

$$
\left[\begin{array}{c}
v_{d h f}^{s} \\
v_{q h f}^{s}
\end{array}\right]=v_{h f}\left[\begin{array}{c}
\cos \left(\omega_{f} t\right) \\
-\sin \left(\omega_{f} t\right)
\end{array}\right]
$$

where $v_{h f}$ is amplitude of the HF voltage, and $\omega_{f}$ is the angular frequency of the HF voltage.

The angular frequency of the injected HF voltage is significantly larger than the rotor angular frequency, and the permanent magnet linkage is significantly smaller than the magnet linkage of the stator current. For these reasons, the permanent magnet linkage can be ignored. Additionally, the stator resistor can be ignored because the stator resistive voltage drop is very small relative to the injected HF voltage. Based on the explanation above, the stator voltage equation is approximated by

$$
\left[\begin{array}{c}
v_{d h f}^{s} \\
v_{q h f}^{s}
\end{array}\right] \approx p\left[\begin{array}{cc}
L-\Delta L \cos \left(2 \theta_{r}\right) & -\Delta L \sin \left(2 \theta_{r}\right) \\
-\Delta L \sin \left(2 \theta_{r}\right) & L+\Delta L \cos \left(2 \theta_{r}\right)
\end{array}\right]\left[\begin{array}{c}
i_{d h f}^{s} \\
i_{q h f}^{s}
\end{array}\right]
$$

In order to estimate the rotor position by using the current generated by the injected HF voltage, the stator current equations are derived as

$$
\begin{aligned}
& {\left[\begin{array}{l}
v_{h f} \sin \left(\omega_{f} t\right) / \omega_{f} \\
v_{h f} \cos \left(\omega_{f} t\right) / \omega_{f}
\end{array}\right]} \\
& =\left[\begin{array}{cc}
L-\Delta L \cos \left(2 \theta_{r}\right) & -\Delta L \sin \left(2 \theta_{r}\right) \\
-\Delta L \sin \left(2 \theta_{r}\right) & L+\Delta L \cos \left(2 \theta_{r}\right)
\end{array}\right]\left[\begin{array}{l}
i_{d h f}^{s} \\
i_{q h f}^{s}
\end{array}\right] \\
& {\left[\begin{array}{l}
i_{d h f}^{s} \\
i_{q h f}^{s}
\end{array}\right]=\frac{v_{h f}}{\omega_{f}\left(L^{2}-\Delta L^{2}\right)}\left[\begin{array}{c}
L \sin \theta_{f}+\Delta L \sin \left(2 \theta_{r}+\theta_{f}\right) \\
L \cos \theta_{f}-\Delta L \cos \left(2 \theta_{r}+\theta_{f}\right)
\end{array}\right]}
\end{aligned}
$$

\subsection{Principle of rotor position estimation}

The conventional HF injection method eliminates the fundamental frequency from the stator current using a BPF. Additionally, an LPF or a notch filter (NF) is used to extract the angular frequency term and the fundamental frequency component. By using a modified synchronous coordinate transformation, however, the number of filters can be reduced. 
As shown in (10), the currents in the stationary frame contain both a fundamental frequency and a high frequency. These frequencies are separated by a coordinate transformation using $2 \hat{\theta}_{r}+\theta_{f}$.

$\left[\begin{array}{l}i_{d h f}^{h} \\ i_{q h f}^{h}\end{array}\right]=\left[\begin{array}{cc}\cos \left(2 \hat{\theta}_{r}+\theta_{f}\right) & \sin \left(2 \hat{\theta}_{r}+\theta_{f}\right) \\ -\sin \left(2 \hat{\theta}_{r}+\theta_{f}\right) & \cos \left(2 \hat{\theta}_{r}+\theta_{f}\right)\end{array}\right]\left[\begin{array}{l}i_{d h f}^{s} \\ i_{q h f}^{s}\end{array}\right]$

$i_{d h f}^{h}=\frac{v_{h f}}{\omega_{f}\left(L^{2}-\Delta L^{2}\right)}\left[L \sin 2\left(\hat{\theta}_{r}+\theta_{f}\right)+\Delta L \sin 2\left(\theta_{r}-\hat{\theta}_{r}\right)\right]$

$i_{q h f}^{h}=\frac{v_{h f}}{\omega_{f}\left(L^{2}-\Delta L^{2}\right)}\left[L \cos 2\left(\hat{\theta}_{r}+\theta_{f}\right)-\Delta L \cos 2\left(\theta_{r}-\hat{\theta}_{r}\right)\right]$

In (11)-(12), $i_{d q h f}^{h}$ is the $\mathrm{d}-\mathrm{q}$ axis current coordinate transformed by $2 \hat{\theta}_{r}+\theta_{f}$.

As a result of the coordinate transformation, the d-q axis currents contain an error term of the rotor position. For this reason, the rotor position can be simply estimated without the heterodyne method or inverse transformation.

Fig. 1 shows the frequency components in the stationary and synchronous reference frame currents, respectively. In Fig. 1(b), the error of the rotor position component is significantly smaller than that of other components. Therefore, this error can be separated from the HF component by the LPF as shown in (13). Hence, it is possible to reduce the delay time caused by the filter compared to the conventional HF signal injection method that uses both the BPF and LPF.

$$
\varepsilon \approx \frac{v_{h f}}{\omega_{f}\left(L^{2}-\Delta L^{2}\right)}\left[\Delta L \sin 2\left(\theta_{r}-\hat{\theta}_{r}\right)\right]
$$

Fig. 2 shows the process of the rotor position estimation.

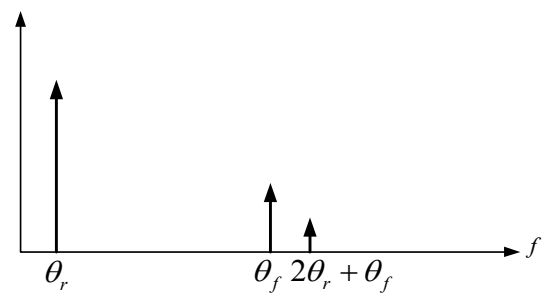

(a)

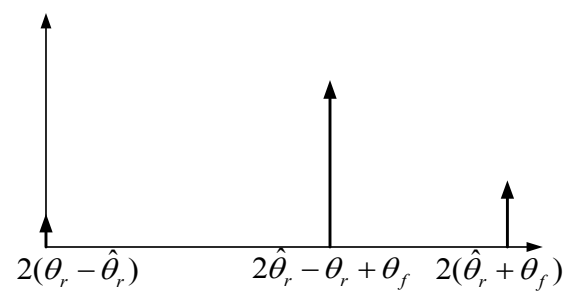

(b)

Fig. 1. The frequency components of the d-q axis current: (a) before transformation by $2 \hat{\theta}_{r}+\theta_{f}$ and (b) after transformation by $2 \hat{\theta}_{r}+\theta_{f}$.

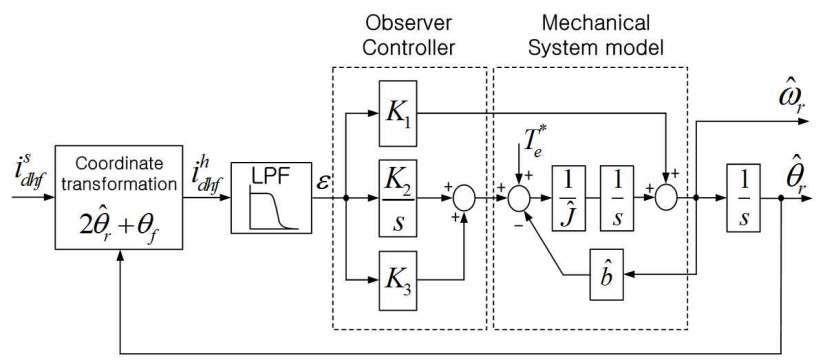

Fig. 2. Block diagram of rotor position estimation using HF signal injection

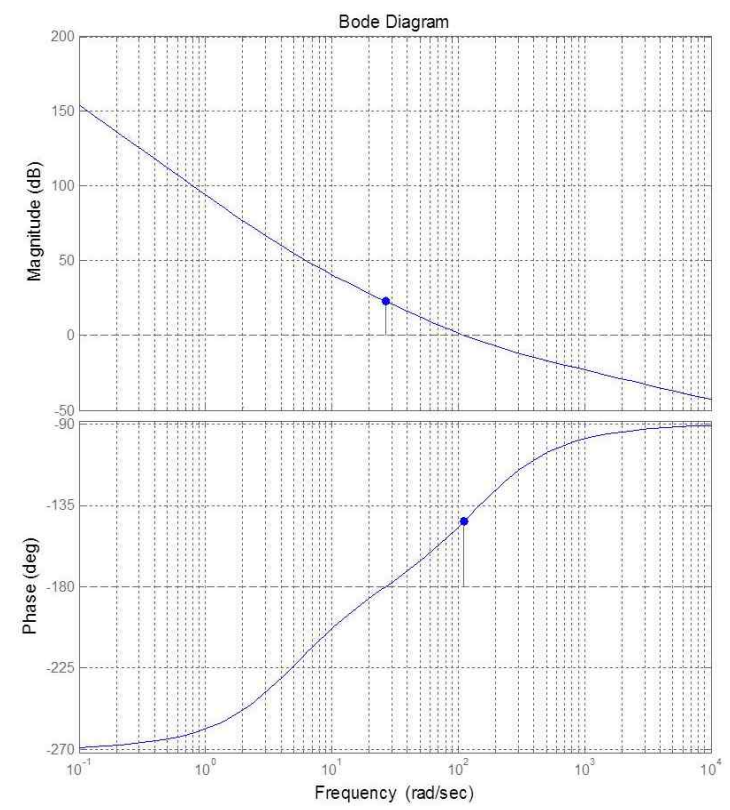

Fig. 3. Frequency response of rotor position estimator

The d-axis current through the LPF is the input of the observer controller. The component of $\sin 2\left(\theta_{r}-\hat{\theta}_{r}\right)$ contained in the $\mathrm{d}$-axis current is controlled to zero by the controller. When the error is controlled to be zero, the rotor position can be estimated [14-16].

To analysis the stability of this estimator, frequency response is plotted as shown in Fig. 3. The gain margin and phase margin are $22.9 \mathrm{~dB}$ and $36.3^{\circ}$, respectively. For this reason, this system can be considered stable.

\section{Full-Order Flux Observer for Sensorless Speed Control}

Because the full-order observer is well known to be a high-performance estimator, it has been used for a wide variety of applications in sensorless control. In this paper, therefore, the full-order observer is used for sensorless control in the medium- and high-speed regions. The physical model of the IPMSM can be regarded as a surface permanent magnet synchronous motor (SPMSM). It can mathematically simplify the full-order observer to decrease 
the computational time. This section reviews the algebraic design of a full-order flux observer for sensorless speed control of an IPMSM for medium- and high-speed operation $[7,8]$.

\subsection{Linear IPMSM model}

The torque of the IPMSM consists of the magnetic torque and the reluctance torque generated by magnetic saliency from the difference in the d-q axis inductance.

$$
T_{e}=\frac{P}{2} \frac{3}{2}\left[\varphi_{f} i_{q}^{r}+\left(L_{d}-L_{q}\right) i_{d}^{r} i_{q}^{r}\right]
$$

Eq. (15) can be derived by (14)

$$
\begin{aligned}
T_{e} & =\frac{P}{2} \frac{3}{2}\left[\varphi_{f} i_{q}^{r}+\left(L_{q}-L_{d}\right) i_{q}^{r}\left(-i_{d}^{r}\right)\right] \\
& =\frac{P}{2} \frac{3}{2}\left[\lambda_{d} i_{q}^{r}+\lambda_{q}\left(-i_{d}^{r}\right)\right]
\end{aligned}
$$

From (15), the rotor-side flux of an IPMSM is expressed as

$$
\boldsymbol{\lambda}=\left[\begin{array}{l}
\lambda_{d} \\
\lambda_{q}
\end{array}\right]=\left[\begin{array}{c}
\varphi_{f} \\
\left(L_{q}-L_{d}\right) i_{q}^{r}
\end{array}\right]
$$

The general model of an IPMSM can be shown in Fig. 4(a). According to the flux model, stator inductance $L_{q}$ is divided into the stator inductance $L_{d}$ and the virtual rotor inductance $L_{q}-L_{d}$. Therefore, Fig. 4(a) can be redrawn as Fig. 4(b) and the physical model of an IPMSM can be regarded as an SPMSM because stator inductances of d-q axis are equivalent. For this reason, the voltage of the IPMSM in the stator reference frame is expressed as follows:

$$
\left[\begin{array}{l}
v_{d}^{s} \\
v_{q}^{s}
\end{array}\right]=\left[\begin{array}{cc}
R_{s}+p L_{d} & 0 \\
0 & R_{s}+p L_{d}
\end{array}\right]\left[\begin{array}{l}
i_{d}^{s} \\
i_{q}^{s}
\end{array}\right]+p\left[\begin{array}{l}
\lambda_{d}^{s} \\
\lambda_{q}^{s}
\end{array}\right]
$$

In (17), a mathematical approximation is not used. It should be noted that the flux model above is insensitive to

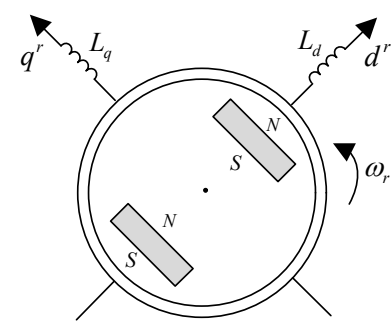

(a)

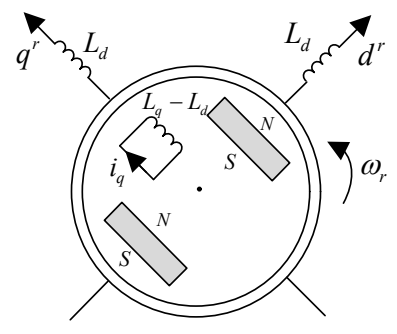

(b)
Fig. 4. Structure of an IPMSM
$L_{q}$ and $\varphi_{f}$, which tends to vary widely depending on the load and thermal condition. This voltage equation is expressed with only $L_{d}[7,8]$.

From the modeling of the IPMSM in (17), the linear state equation of the IPMSM in the stator reference frame is expressed as follows [9]:

$$
p \mathbf{x}=\left[\begin{array}{cc}
-\frac{R_{s}}{L_{d}} \mathbf{I} & -\mathbf{J} \frac{\omega_{r}}{L_{d}} \\
\mathbf{0} & \mathbf{J} \omega_{r}
\end{array}\right] \mathbf{x}+\left[\begin{array}{c}
\mathbf{I} / L_{d} \\
\mathbf{0}
\end{array}\right] \boldsymbol{u}
$$

where, $\mathbf{x}=\left[\begin{array}{llll}i_{d}^{s} & i_{q}^{s} & \lambda_{d}^{s} & \lambda_{q}^{s}\end{array}\right], \quad \mathbf{u}=\left[\begin{array}{ll}v_{d}^{s} & \nu_{q}^{s}\end{array}\right]$

$$
\mathbf{I}=\left[\begin{array}{ll}
1 & 0 \\
0 & 1
\end{array}\right], \quad \mathbf{J}=\left[\begin{array}{cc}
0 & -1 \\
1 & 0
\end{array}\right], \quad \mathbf{0}=\left[\begin{array}{ll}
0 & 0 \\
0 & 0
\end{array}\right] .
$$

\subsection{Full-order observer for flux estimation}

A full-order observer for flux estimation of the IPMSM is constructed on the basis of (18):

$$
\begin{aligned}
p \hat{\mathbf{x}}= & {\left[\begin{array}{cc}
-\frac{R_{s}}{L_{d}} \mathbf{I} & -\mathbf{J} \frac{\hat{\omega}_{r}}{L_{d}} \\
\mathbf{0} & \mathbf{J} \hat{\omega}_{r}
\end{array}\right] \hat{\mathbf{x}}+\left[\begin{array}{c}
\mathbf{I} / L_{d} \\
\mathbf{0}
\end{array}\right] \mathbf{u} } \\
& +\left[\begin{array}{c}
G_{1} \mathbf{I}+G_{2} \mathbf{J} \\
H_{1} \mathbf{I}+H_{2} \mathbf{J}
\end{array}\right]\left[\begin{array}{ll}
\mathbf{I} & \mathbf{O}
\end{array}\right][\hat{\mathbf{x}}-\mathbf{x}]
\end{aligned}
$$

where G1, G2, H1, and H2 are the observer feedback gains.

Obtaining these observer gains is complicated because the observer is a four-dimensional system. Therefore, using the principle of complex vector notation, we simply express the observer equation as [8]

$$
\begin{aligned}
p\left[\begin{array}{l}
\hat{\boldsymbol{i}} \\
\hat{\lambda}
\end{array}\right] & =\left[\begin{array}{cc}
-\frac{R_{s}}{L_{d}} & -\frac{\boldsymbol{j} \hat{\omega}_{r}}{L_{d}} \\
\mathbf{0} & \boldsymbol{j} \hat{\omega}_{r}
\end{array}\right]\left[\begin{array}{l}
\hat{\boldsymbol{i}} \\
\hat{\lambda}
\end{array}\right]+\left[\begin{array}{c}
1 / L_{d} \\
0
\end{array}\right] \boldsymbol{v}+\left[\begin{array}{c}
G_{1}+\boldsymbol{j} G_{2} \\
H_{1}+\boldsymbol{j} H_{2}
\end{array}\right](\hat{\boldsymbol{i}}-\boldsymbol{i}) \\
& =\boldsymbol{A}\left[\begin{array}{l}
\hat{\boldsymbol{i}} \\
\hat{\lambda}
\end{array}\right]+\boldsymbol{B} \boldsymbol{v}+\boldsymbol{H}(\hat{\boldsymbol{i}}-\boldsymbol{i})
\end{aligned}
$$

Fig. 5 shows the block diagram of the full-order flux observer. The characteristic equation of the full-order observer in (20) is expressed as

$$
|s \boldsymbol{I}-\boldsymbol{A}-\boldsymbol{H C}|=\left(s+\frac{R}{L_{d}}-H_{1}-\boldsymbol{j} H_{2}\right)\left(s-\boldsymbol{j} \hat{\omega}_{r}\right)+\frac{\boldsymbol{j} \hat{\omega}_{r}}{L_{d}}\left(G_{1}+\boldsymbol{j} G_{2}\right)
$$

In addition, when the above system has two certain poles, the characteristic equation is expressed as

$$
\begin{aligned}
& \left(s-\left(\alpha_{1}+j \beta_{1}\right)\right)\left(s-\left(\alpha_{2}+j \beta_{2}\right)\right) \\
& \quad=s^{2}-\left(\alpha_{1}+j \beta_{1}+\alpha_{2}+j \beta_{2}\right) s+\left(\alpha_{1}+j \beta_{1}\right)\left(\alpha_{2}+j \beta_{2}\right)
\end{aligned}
$$




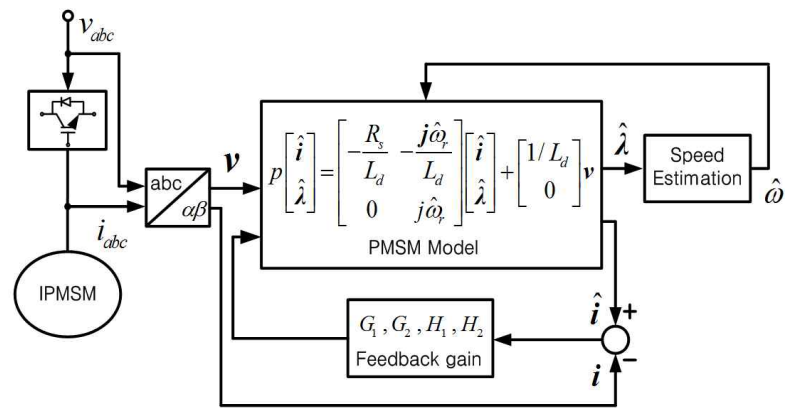

Fig. 5. Block diagram of the full-order flux observer

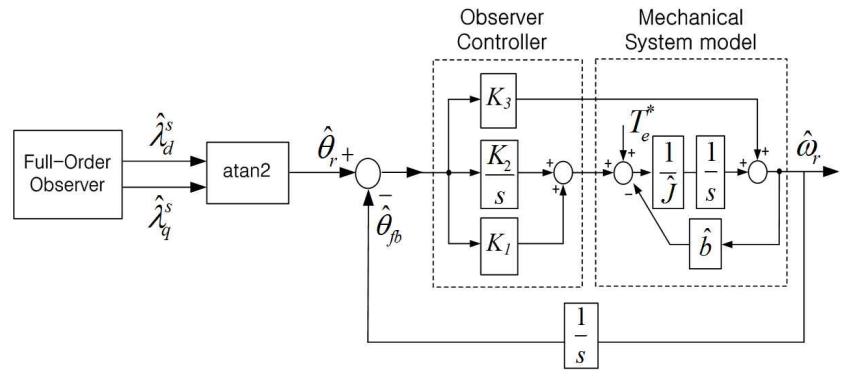

Fig. 6. Block diagram of the rotor position and speed estimation

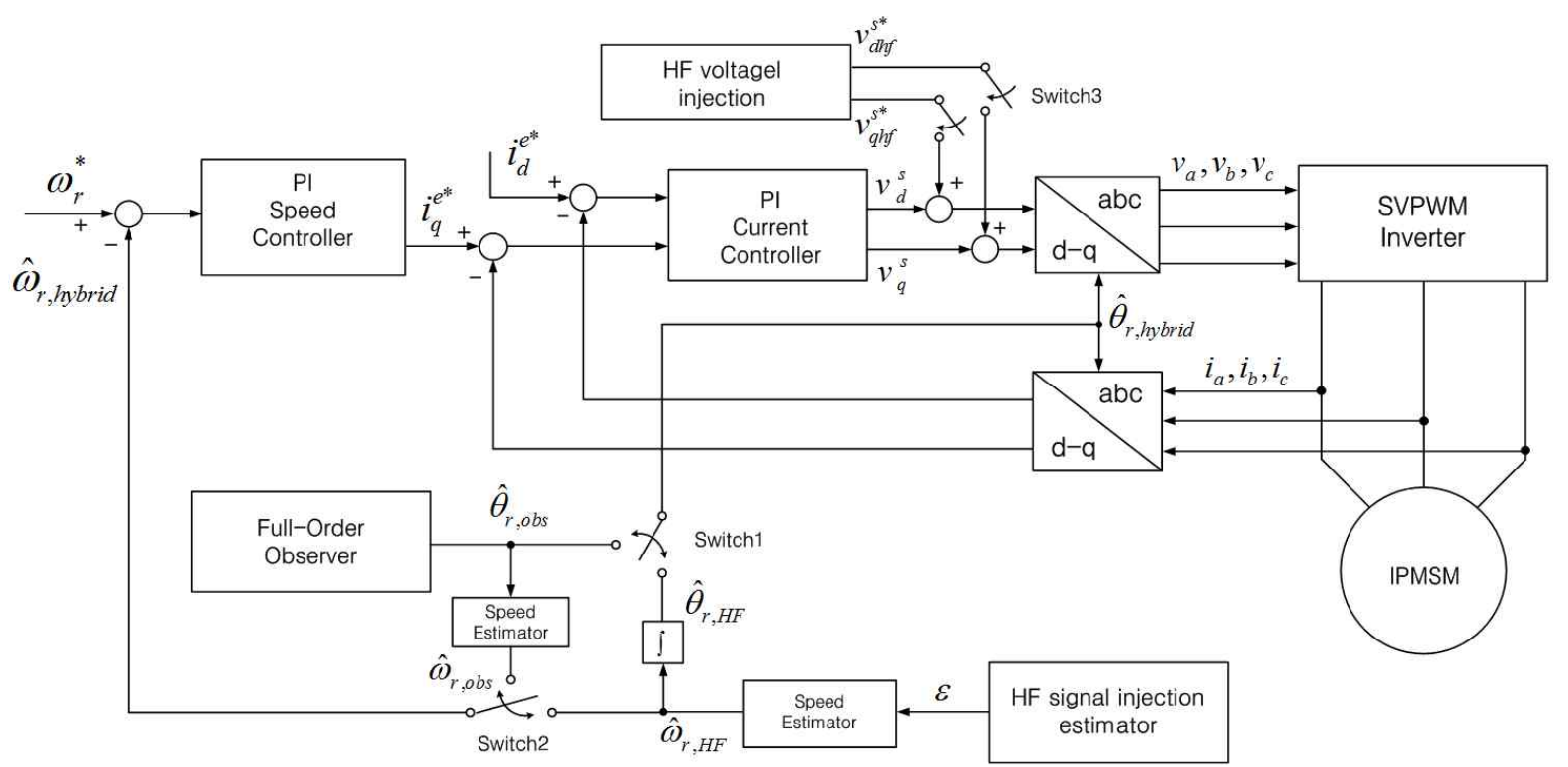

Fig. 7. Overall structure of the proposed hybrid sensorless control system

Hence, comparing (21) with (22), observer gains can be expressed as

$$
\begin{aligned}
& H_{1}=\frac{R}{L_{d}}+\alpha_{1}+\alpha_{2}, \\
& H_{2}=-\hat{\omega}_{r}+\beta_{1}+\beta_{2} \\
& G_{1}=-\left(\alpha_{1}+\alpha_{2}\right) L_{d}+\left(\alpha_{1} \beta_{2}+\beta_{1} \alpha_{2}\right) \frac{L_{d}}{\hat{\omega}_{r}}, \\
& G_{2}=\left(\hat{\omega}_{r}-\left(\beta_{1}+\beta_{2}\right)\right) L_{d}-\frac{L_{d}}{\hat{\omega}_{r}}\left(\alpha_{1} \alpha_{2}-\beta_{1} \beta_{2}\right)
\end{aligned}
$$

Fig. 6 shows the process of rotor position and speed estimation using a full-order observer. The rotor position can be computed with the estimated stator flux. On the other hand, the process of rotor speed estimation is the same as in Section II, but the observer controller gains (K1, $\mathrm{K} 2$, and K3) must choose a different value. These gains have been chosen with a trial-and-error procedure to get the best tradeoff between stability and convergence time of observer. The integral value of the estimated rotor speed is a negative feedback term which makes the observer controller input with estimated rotor position by full-order observer.

\section{Simple Strategy of the Hybrid System}

Thus far, we have introduced two types of sensorless methods used in the hybrid system. A simple method of HF signal injection was introduced in Section II. This method is intended to be operated in the low-speed region. On the other hand, the full-order observer introduced in Section III has problems during low-speed operation because of the low current and voltage magnitudes and inaccurate parameters. However, the full-order observer exhibits high performance during medium- and high-speed operation. Hence, each of these methods has difficulty obtaining high performance throughout the entire speed range.

In this section, a hybrid system is proposed by combining the HF signal injection method and a full-order observer to cover the entire speed range from zero speed to high speed. Conventional methods of hybrid systems change another observer by using speed-dependent transition function to get the stable transition [17-21]. They have 
good performance but the switching point is ambiguous. Therefore, it should be determine by trial and error to guarantee stable transition. However, the switching point can be variable according to load condition and performance of both estimators. Hence, in this method, it should delay the switching point to ensure the stable transition that leads increasing the time to use the HF injection method in low speed region. As a result, optimal switching point is necessary to stable and fast transition.

The proposed hybrid method determines the optimal switching point by using the error signal of both estimators. This method does not have to consider the load condition and performance of both estimators, and only selects the proper range of error that leads to a stable transition.

\subsection{From a standstill to high-speed operation}

At initial operation, the estimated rotor position $\hat{\theta}_{r, H F}$ from HF signal injection is used to operate the motor. At the same time, a full-order observer only estimates the rotor position $\hat{\theta}_{r, o b s}$. When the error of these estimated values is close to zero, sensorless control is performed by using a full-order observer, and the HF signal is eliminated.

$$
\tilde{\theta}_{\text {err }}=\hat{\theta}_{r, H F}-\hat{\theta}_{r, o b s}<k
$$

The range of the error has to be set close to zero. A smaller value of $\mathrm{k}$ can be a stable transition. As the value of $\mathrm{k}$ decreases, however, the range of HF signal injection increases, which causes acoustical noise and signal injection losses. Therefore, to reduce the HF signal injection range and guarantee a stable transition, the proper value of $\mathrm{k}$ should be experimentally chosen. For a more stable transition, the system counts the instances when entering within the range of error and makes a transition when a certain error count $(\mathrm{N})$ is exceeded. The switching point varies according to $\mathrm{k}$ and the performance of the full-order observer at low speed. This hybrid strategy is presented as

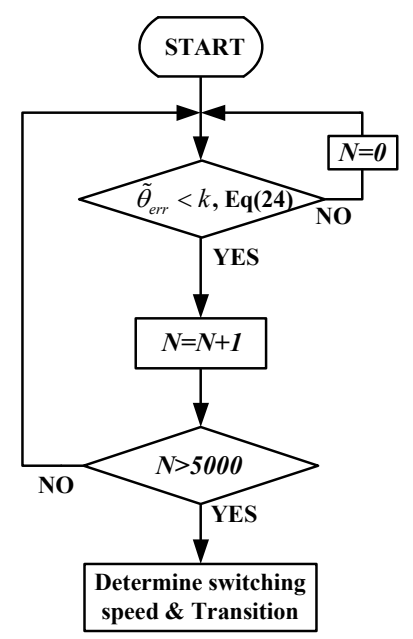

Fig. 8. Flow chart for standstill to high-speed operation flow chart in Fig. 8.

\subsection{From High-speed operation to a standstill}

As mentioned previously, the switching point when the mode transition occurs from a low speed to a high speed becomes the switching point of the entire hybrid system. As a result, the transition occurs at this point when it moves back from high speed to the low speed. In mediumand high-speed operation, however, the error of two estimators cannot be used anymore because the HF signal was eliminated. For this reason, the transition to HF signal injection estimation is not performed smoothly at the switching point. Before reaching the switching point, an HF signal is injected in advance to solve this problem. Additionally, when making a transition in the mode at the switching point, the initial value of the HF injection estimator has to receive the value of the full-order observer for usage.

$$
\hat{\theta}_{r, H F}^{s w}=\hat{\theta}_{r, o b s}^{s w}
$$

Unlike the previous method, even if a mode transition occurs, the full-order observer continues to estimate.

The overall structure of the proposed hybrid sensorless control system for the IPMSM drive is presented in Fig. 7.

\section{Experimental Results}

In order to verify the proposed hybrid system for sensorless control, experiments were carried out by using the 11-kW IPMSM in Fig. 9. The load is a $15-\mathrm{kW}$

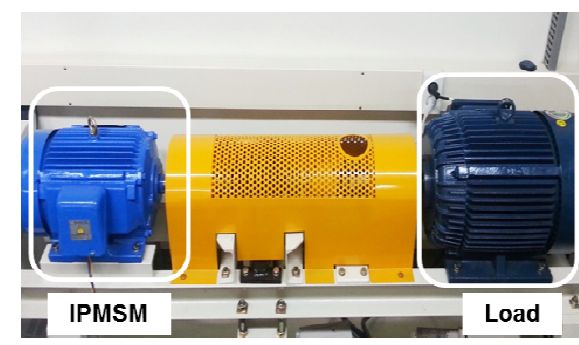

(a) $11 \mathrm{~kW}$ IPMSM with Load

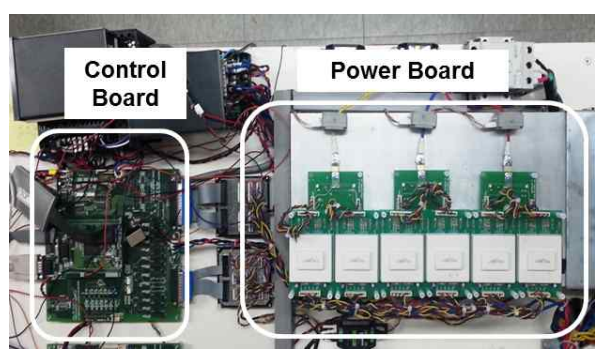

(b) Control board and Power board

Fig. 9. Experimental setup of the IPMSM drive system 
Table 1. Motor parameters

\begin{tabular}{c|c}
\hline Stator Resistance & $0.349 \Omega$ \\
\hline d-axis Inductance & $13.16 \mathrm{mH}$ \\
\hline q-axis Inductance & $15.6 \mathrm{mH}$ \\
\hline Number of Poles & 6 \\
\hline Flux Linkage & $0.554 \mathrm{~Wb}$ \\
\hline Rated Power & $11 \mathrm{~kW}$ \\
\hline Rated Current & $19.9 \mathrm{~A}$ \\
\hline Rated Speed & $1650 \mathrm{rpm}$ \\
\hline
\end{tabular}

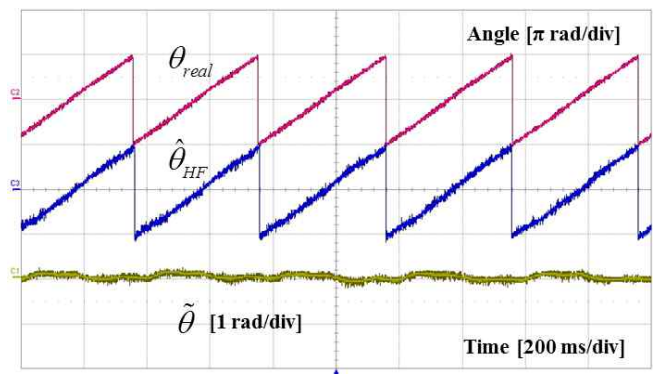

(a)

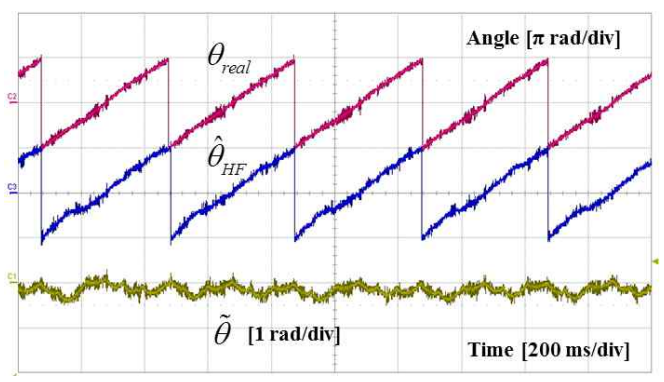

(b)

Fig. 10. Experimental results at low speed (50 rpm): (a) proposed HF signal injection method; (b) Fullorder observer

induction motor. The switching frequency and sampling period $T_{s}$ are $10 \mathrm{kHz}$ and $100 \mu \mathrm{s}$, respectively. The IPMSM parameters for the experiment are presented in Table 1. In this experiment, the range of error is set to $k=0.2[\mathrm{rad}]$ found via trial-and-error to obtain the best tradeoff between a fast and stable transition. In addition, $N$ is set to 5000 .

Fig. 10 shows the performance of rotor position estimation to compare two methods at low speed operation (50rpm). The estimated rotor position is more stable when using the proposed method than using full-order observer.

Fig. 11 shows the low speed control using proposed HF signal injection method. When a full step load torque is applied to the system, the speed quickly reaches steady state region. From these experimental results, the performance of proposed method can be verified at low speed operation. Further, it is possible to know that in order to obtain good performance in all speed range, hybrid method is needed.

The estimated rotor positions from the two methods are shown in Fig. 12. At the beginning of the IPMSM drives, it

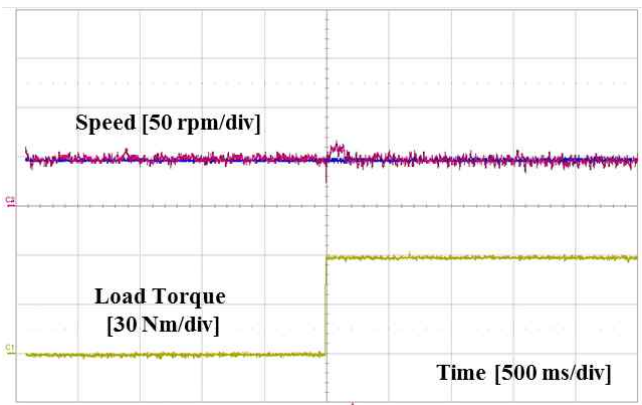

Fig. 11. Low speed control using the proposed HF signal injection method with step full load torque

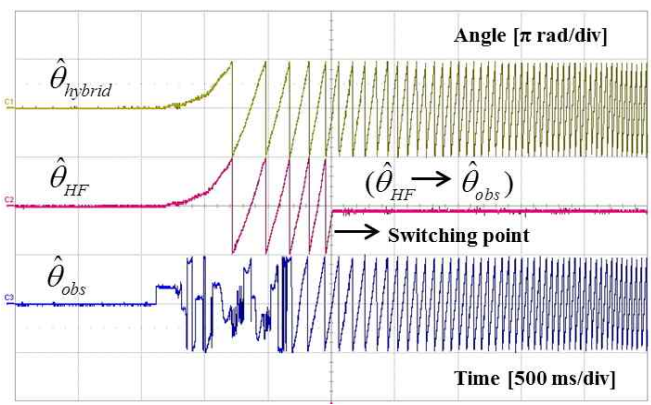

Fig. 12. Hybrid rotor position estimation with the two methods

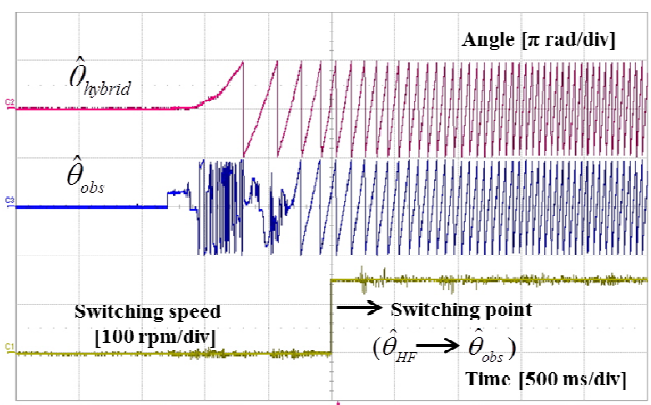

Fig. 13. Hybrid rotor position estimation and switching speed

is observed that the hybrid rotor position corresponds with the rotor position obtained by HF signal injection. On the other hand, rotor position obtained by a full-order observer is not estimated. As the value of the full-order observer begins to provide a good estimate, the hybrid rotor position is switched to the rotor position obtained by a full-order observer. At the same time, it is observed that HF signal injection is eliminated. In addition, it is verified that a smooth mode transition occurs by looking at the hybrid rotor position.

Fig. 13 shows the switching speed when the transition is implemented. The switching speed is always slightly different because the performance of the full-order observer at startup is also always different, as can be seen by comparison with Fig. 12. The transition is performed in the vicinity of $150 \mathrm{rpm}$, which is $9 \%$ of the rated speed for the 
parameter values used in this experiment.

Fig. 14 shows the performance of the proposed hybrid sensorless speed control from a standstill to the rated

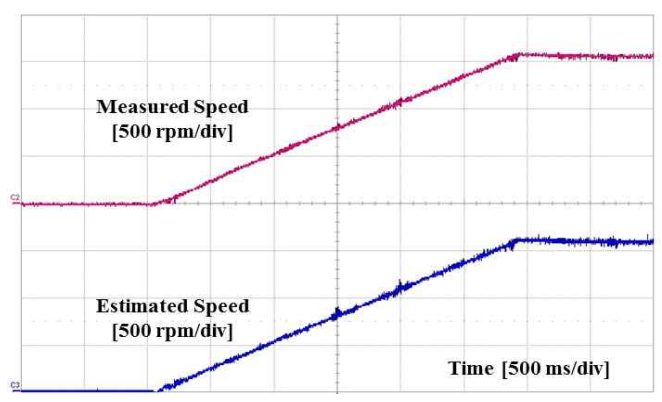

(a)

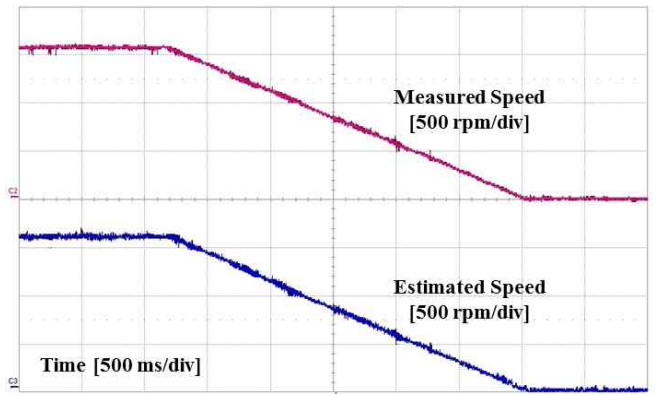

(b)

Fig. 14. Hybrid sensorless speed control under light load : measured rotor speed (red) and estimated rotor speed (blue)

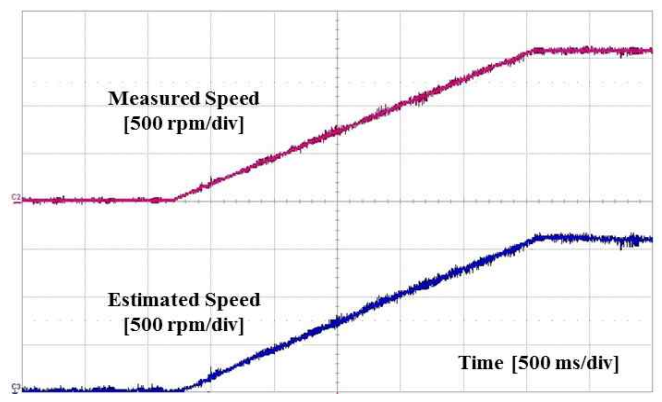

(a)

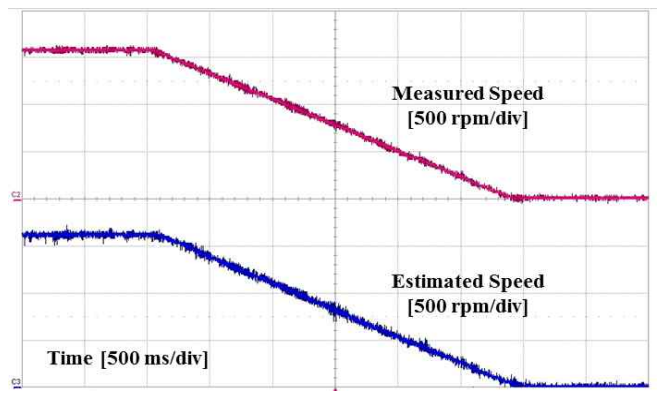

(b)

Fig. 15. Hybrid sensorless speed control under full load : measured rotor speed (red) and estimated rotor speed (blue) speed (1650 rpm) under light load. Good performance is observed for a wide speed range because the estimated and measured speeds are matched to each other. Additionally, the transition between each method is well conducted when the speed increases and decreases.

In comparison with the light-load condition, it can be verified that the control performs well from low speed to the rated speed under the full-load condition, as shown in Fig. 15. It is observed that the estimated value and the actual value are almost identical. It is also observed that the speed is well controlled when it decreases from a high speed to zero speed. Additionally, it is observed that the rotor position estimated by the proposed HF signal injection method is well estimated at startup, as shown in Figs. 14(a) and 15(a).

Fig. 16 shows the performance of sensorless speed reversal from -500 to $500[\mathrm{rpm}]$ (low and middle speed region). In both directions, smooth transition to low and middle speed region can be seen in above figure. For more detailed information at zero and low speed region, an expanded figure can be seen in below. The estimation error of the rotor position is very small during the speed reversal.

To compare proposed method to conventional method, additional experimental result is provided as shown in Fig. 17. As stated previously in Section VI, conventional hybrid methods use the speed-dependent transition function to get the smooth transition between estimation methods. The interval of transition between each method is set to 100 and $200[\mathrm{rpm}]$ which are $6 \% \sim 12 \%$ of rated speed.

It can be seen that estimated rotor position by full order observer does not reach the steady state in transition interval. Therefore, hybrid rotor position and waveform of rotor speed have distortions. In this method, it should delay the switching point according to the performance of estimator. On the other hand, in the case of using the proposed method, since the transition occurs after the

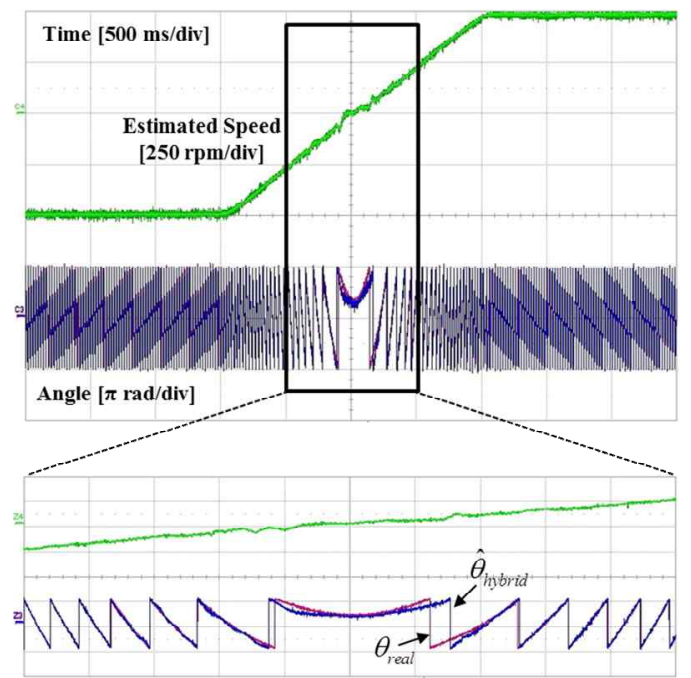

Fig. 16. Experimental result of speed reversal under the light load 
reaching the steady-state regardless of the estimated speed of the full-order observer, it can be determined to be the most stable and optimal switching point.

\section{Conclusion}

This paper proposes a simple HF signal injection method and a hybrid system for sensorless speed control of an IPMSM. The proposed methods decrease the number of filters, and it is possible to operate over the entire speed range. In particular, the hybrid method compensates for the weaknesses of the observer-based method, which does not have good performance at start-up and in the low-speed range. The experimental results demonstrate that the proposed methods effectively realize a feasible hybrid system for sensorless speed control.

\section{Acknowledgements}

This work was supported by the Hyosung Power \& Industrial Systems Performance Group.

\section{References}

[1] R. Wu, "Permanent magnet motor drive without a shaft sensor," IEEE Trans. Industry Applications, vol. 27, no. 5, pp. 1005-1011, Sep/Oct 1991.

[2] J. I. Ha, S. J. Kang, and S. K. Sul, "Positioncontrolled Synchronous Reluctance Motor Without Rotational Transducer," IEEE Trans. Industry Applications, vol. 35, no. 6, pp. 1393-1398. Nov/Dec 1999.

[3] B. Nahid-Mobarakeh, F. Meibody-Tabar, and F.-M. Sargos, "Back EMF Estimation-Based Sensorless Control of PMSM: Robustness With Respect to Measurement Errors and Inverter Irregularities," IEEE Trans. Industry Applications, vol. 43, no. 2, pp. 485-494, Mar / Apr. 2007.

[4] Z. Wang, K. Lu, and F. Blaabjerg, "A Simple Startup Strategy Based on Current Regulation for BackEMF-Based Sensorless Control of PMSM," IEEE Trans. Power Electronics, vol. 27, no. 8, pp.38173825, Aug. 2012.

[5] K. B. Lee and F. Blaabjerg, "Reduced Order Extended Luenberger Observer Based Sensorless Vector Control Driven by Matrix Converter With Non-Linearity Compensation," IEEE Trans. Industrial Electronics, vol. 53, no. 1, pp. 66-75, Feb. 2006.

[6] S. Bolognani, R. Oboe, and M. Zigliotto, "Sensorless Full-Digital PMSM Drive with EKF Estimation of Speed and Rotor Position," IEEE Trans. Industrial Electronics, vol. 46, no. 1, pp. 184-191, Feb. 1999.

[7] A. Matsumoto, M. Hasegawa, and K. Matsui, "A Novel Flux Model and Decoupling Control on Maximum Torque Control Frame for IPMSM Position
Sensorless Vector Control,” Proc. SPEEDAM 2010, pp. 312-317, Jun. 2010.

[8] A. Matsumoto, M. Hasegawa, M. Tomita, and K. Matsui, "Algebraic Design of Full-Order Flux Observer for IPMSM Position Sensorless Control," in conf. IEEE / IEMDC conf, pp. 1276-1281, May. 2011.

[9] S. Pongam and S. Sangwongwanich, "Stability and Dynamic Performance Improvement of Adaptive Full-Order Observers for Sensorless PMSM Drive", IEEE Trans. Power Electronics, vol. 27, no. 2, pp. 588-600, Feb. 2012.

[10] K. B. Lee and F. Blaabjerg, "An Improved DTCSVM Method for Sensorless Matrix Converter Drives Using an Overmodulation and Simple NonLinearity compensation," IEEE Trans. Industrial Electronics, vol. 54, no. 6, pp. 3155-3166, Dec. 2007.

[11] K. B. Lee, F. Blaabjerg, and T. W. Yoon, "Speed Sensorless DTC-SVM for Matrix Converter Drives with Simple Non-Linearity Compensation," IEEE Trans. Industry Applications, vol. 43, no. 6, pp. 16391649, Nov./Dec. 2007.

[12] J. Holtz, "Sensorless Control of Induction Machineswith or Without Signal Injection?," IEEE Trans. Industrial Electronics, vol. 53, no. 1, pp. 7-30, Feb. 2006.

[13] J. H. Jang, J. I. Ha, M. Ohto, K. Ide, and S. K. Sul, "Analysis of Permanent-Magnet Machine for Sensorless Control Based on High-Frequency Signal Injection," IEEE Trans. Industry Application, vol. 40, no. 6, pp. 1595-1604, Nov. 2004.

[14] P. L Jansen and R. D. Lorenz, "Transducerless Field Orientation Concepts Employing SaturationInduced Saliencies in Induction Machines," IEEE Trans. Industry Applications, vol. 32, no. 6, pp. 1380-1392, Nov / Dec 1996.

[15] M. J. Corley and R. D. Lorenz, "Rotor Position and Velocity Estimation for a Salient-Pole Permanent Magnet Synchronous Machine at Standstill and High Speeds," IEEE Trans. Industry Applications, vol. 27, no. 4, pp. 784-789, Jul / Aug. 1998.

[16] R. D. Lorenz and K. W. Van Patten, "High-resolution velocity estimation for all-digital, AC servo drives," IEEE Trans. Industry Applications, vol. 27, no. 4, pp. 701-705. Jul / Aug 1991.

[17] C. Silva, G. M. Asher, and M. Sumner, "Hybrid Rotor Position Observer for Wide Speed-Range Sensorless PM Motor Drives Including Zero Speed," IEEE Trans. Industrial Electronics, vol. 53, no. 2, pp. 373-378, Apr. 2006.

[18] G. D. Andreescu, C.I. Pitic, F. Blaabjerg, and I. Boldea, "Combined Flux Observer with Signal Injection Enhancemnet for Wide Speed Range Sensorless Direct Torque Control of IPMSM Drives," IEEE Trans. Energy conversion, vol. 23, no. 2, pp. 393-402, Jun. 2008.

[19] A. Piippo and J. Luomi, "Adaptive Observer Combined with HF Signal Injection for Sensorless Control of PMSM Drives," in Proc. IEMDC 2005, 
San Antonio, TX, pp. 674-681.

[20] G. Foo and M. F. Rahman, "Sensorless Sliding-Mode MTPA Control of an IPM Synchronous Motor Drive Using a Sliding-Mode Observer and HF Signal Injection," IEEE Trans. Industrial Electronics, vol. 57, no. 4, pp. 1270-1278, Apr. 2010.

[21] O. Wallmark and L. Harnefors, "Sensorless Control of Salient PMSM Drives in the Transition Region," IEEE Trans. Industrial Electronics, vol. 53, no. 4, pp. 1179-1187, Aug. 2009.

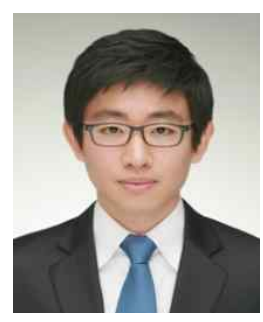

Hyun-Woo Sim received the B.S. degree in Electrical and Computer Engineering from Ajou University, Suwon, Korea, in 2013. He is currently working toward the M.S degree at Ajou University, Suwon, Korea. His research interests include electric machine drives and multilevel inverter and reliability.

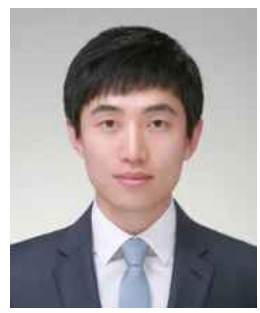

June-Seok Lee received the B.S. and M.S. degrees in Electrical and Computer Engineering from the Ajou University, Korea, in 2011 and 2013, respectively. $\mathrm{He}$ is currently working toward the Ph.D. degree at Ajou University, Korea. His research interests include grid-connected systems, multilevel inverter and reliability.

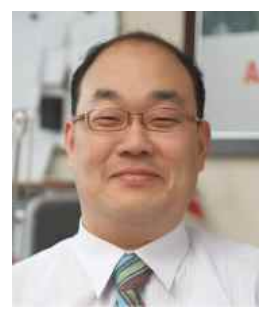

Kyo-Beum Lee received the B.S. and M.S. degrees in electrical and electronic engineering from the Ajou University, Korea, in 1997 and 1999, respectively. He received the Ph.D. degree in electrical engineering from the Korea University, Korea in 2003. From 2003 to 2006 , he was with the Institute of Energy Technology, Aalborg University, Aalborg, Denmark. From 2006 to 2007, he was with the Division of Electronics and Information Engineering, Chonbuk National University, Jeonju, Korea. In 2007 he joined the School of Electrical and Computer Engineering, Ajou University, Suwon, Korea. He is an associated editor of the IEEE Transactions on Power Electronics and the Journal of Power Electronics. His research interests include electric machine drives, renewable power generations, and electric vehicles. 